\title{
TUTTE POLYNOMIALS AND LINK POLYNOMIALS
}

\author{
FRANÇOIS JAEGER
}

(Communicated by Thomas H. Brylawski)

\begin{abstract}
We show how the Tutte polynomial of a plane graph can be evaluated as the "homfly" polynomial of an associated oriented link. Then we discuss some consequences for the partition function of the Potts model, the Four Color Problem and the time complexity of the computation of the homfly polynomial.
\end{abstract}

1. Introduction. Recently several new invariants of knots and links have been discovered (see [12] for a survey) and proved to be quite useful for the solution of difficult problems (see for instance $[\mathbf{1 3}, \mathbf{1 6}, \mathbf{1 9}]$, which deal with the crossing number of alternating links). These invariants are polynomials which can be defined in a purely combinatorial way on link diagrams. At the same time they can be defined (via representations of the braid groups) as traces in certain algebras (see [11]). We shall be concerned here with the "homfly" invariant (whose name comes from the initials of the authors of [7]), which contains as special cases the Jones polynomial introduced in [10] and the Alexander-Conway polynomial [5].

As noted in [6], as combinatorial objects the new polynomial invariants exhibit a striking similarity with the Tutte polynomials [21] of plane graphs: they satisfy analogous local reduction properties which allow their recursive computation. In fact Thistlethwaite [19], using results of Kauffman [13], obtained a spanning tree expansion for the Jones polynomial which is essentially the same as the one obtained by Tutte for his polynomial. An interesting corollary is that the Jones polynomial of an alternating oriented link can be evaluated as a Tutte polynomial (with one free variable) of an associated plane graph. This work has been extended by Kauffman [12] who defines a "bracket" polynomial of which both Tutte polynomials and Jones polynomials are special cases.

On the algebraic side, Jones $[\mathbf{1 0}, \mathbf{1 1}]$ pointed out a close correspondence between his polynomial and the Potts model of statistical mechanics (see [2, Chapter 12]): the algebra used by physicists to study the partition function of the Potts model [18] is the framework for the definition of the Jones polynomial. This correspondence is further explored by Kauffman [14]. Since the partition function of the Potts model is just another version of the Tutte polynomial, we have again a deep connection between the Jones and Tutte polynomials.

The purpose of this note is to show that the Tutte polynomial of a plane graph can be computed as a "homfly" polynomial of an associated link. Thus the Four Color problem and the problem of an exact solution of the Potts model can be viewed as problems about links, and in particular as problems about closed braids.

Received by the editors April 20, 1987.

1980 Mathematics Subject Classification (1985 Revision). Primary 57M25, 05C10; Secondary 57M15, 05C15. 
2. Tutte polynomials and homfly polynomials. The reader is referred to $[\mathbf{3}, \mathbf{4}, \mathbf{1 5}]$ for terminology.

The Tutte polynomial (or dichromate) of a (nonempty) graph $G$, introduced in $[\mathbf{2 0}, \mathbf{2 1}]$, is a polynomial in two variables $t(G, x, y)$ which satisfies the following properties:

(1) If $G$ has exactly one edge, $t(G, x, y)=x$ if this edge is a bridge and $t(G, x, y)=$ $y$ if this edge is a loop.

(2) If $e$ is an edge of $G$ which is neither a bridge nor a loop:

$$
t(G, x, y)=t(G-e, x, y)+t(G . e, x, y)
$$

where $G-e$ (respectively, G.e) denotes the graph obtained from $G$ by deleting (respectively, contracting) $e$.

(3) If $G$ has at least two edges and $e$ is a bridge (respectively, loop) of $G$, $t(G, x, y)=x t(G . e, x, y)$ (respectively, $t(G, x, y)=y t(G-e, x, y)$ ).

Note that properties (1), (2), (3) allow the computation of $t(G, x, y)$ for any graph $G$. The consistency of (1), (2), (3) considered as defining axioms follows from the existence of the following subgraph expansion for $t(G, x, y)$ :

$$
t(G, x, y)=\sum_{F \subseteq E(G)}(x-1)^{c(F)-c(E(G))}(y-1)^{|F|-|V(G)|+c(F)},
$$

where $c(F)$ denotes the number of connected components of the graph formed on the vertices of $G$ by the edges of $F$.

We now define the homfly invariant [7] of a tame oriented link. One can associate to every oriented link diagram $L$ a homogeneous Laurent polynomial of degree zero in three variables which we denote by $P(L, x, y, z)$ in such a way that the following properties hold:

(i) $P(L, x, y, z)=1$ if $L$ corresponds to the trivial knot.

(ii) If $L^{+}, L^{-}, L^{\circ}$ are three diagrams which coincide except inside a small disk where they behave as depicted in Figure 1 then $x P\left(L^{+}, x, y, z\right)+y P\left(L^{-}, x, y, z\right)+$ $z P\left(L^{\circ}, x, y, z\right)=0$.

(iii) If $L$ and $L^{\prime}$ correspond to isotopic oriented links, $P(L, x, y, z)=P\left(L^{\prime}, x, y, z\right)$.

The existence of $P$ can be proved either combinatorially by working on diagrams only, or by algebraic methods which involve the representation of links as closed braids.

Another property of $P$ which will be useful is the following (see for instance [9, properties 7 and 8, pp. 314-315]):

(iv) If $L$ is split into $L_{1}$ and $L_{2}$, then

$$
P(L, x, y, z)=(-(x+y) / z) P\left(L_{1}, x, y, z\right) P\left(L_{2}, x, y, z\right)
$$
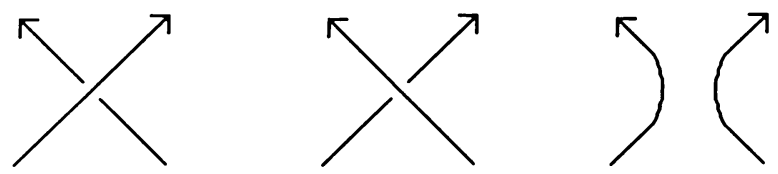

FIGURE 1 

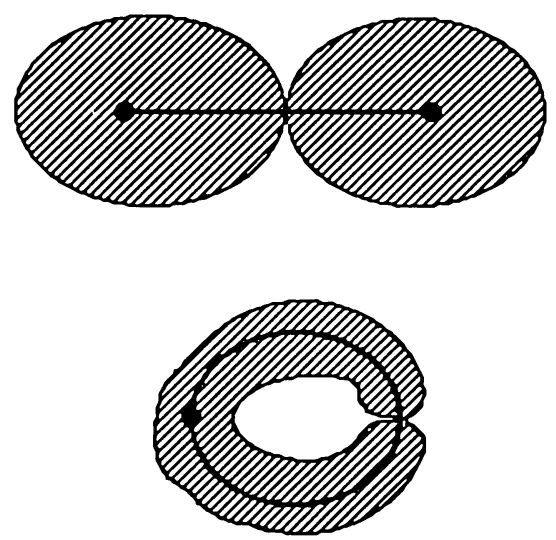

FIGURE 2

If $L$ is the connected sum of $L_{1}$ and $L_{2}$, then

$$
P(L, x, y, z)=P\left(L_{1}, x, y, z\right) P\left(L_{2}, x, y, z\right) .
$$

Let us consider now a connected plane graph $G$. We associate to $G$ a link diagram $D(G)$ as follows. First we construct the medial graph $M(G)$ of $G$ (see $[17$, p. 47]). $M(G)$ is a 4-regular plane graph (a universe in the terminology of [15]) from which $G$ can be recovered in the following way: color the faces of $M(G)$ in two colors (say black and white) in such a way that adjacent faces have different colors; then $G$ has one vertex $v(f)$ in the interior of each black face $f$ of $M(G)$, and one edge with ends $v\left(f_{1}\right), v\left(f_{2}\right)$ for each vertex of $M(G)$ incident to the black faces $f_{1}, f_{2}$. This construction is displayed in Figure 2 for the connected plane graphs with one edge.

Now to obtain $D(G)$ we first direct every edge of $M(G)$ in such a way that the incident black face is on its left, and then we replace the neighborhood of each vertex by a diagram as depicted in Figure 3 .

An example of the construction of $D(G)$ is given in Figure 4 ( $G$ is the complete graph on 4 vertices).

Note that to each face of $G$ corresponds an unknotted component of $D(G)$ which is oriented clockwise (with the exception of the infinite face). Each edge of $G$ defines a certain interweaving of the components associated to the adjoining faces, and $D(G)$ is clearly alternating.

We are now ready to state and prove the following result.
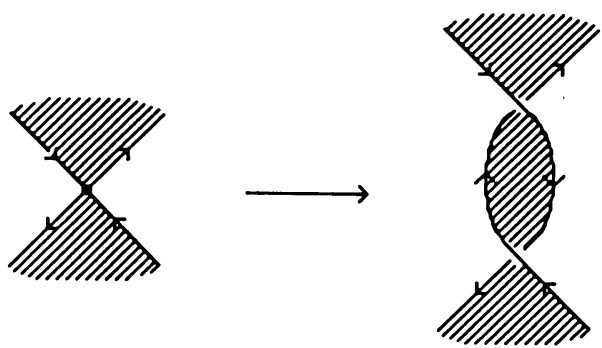


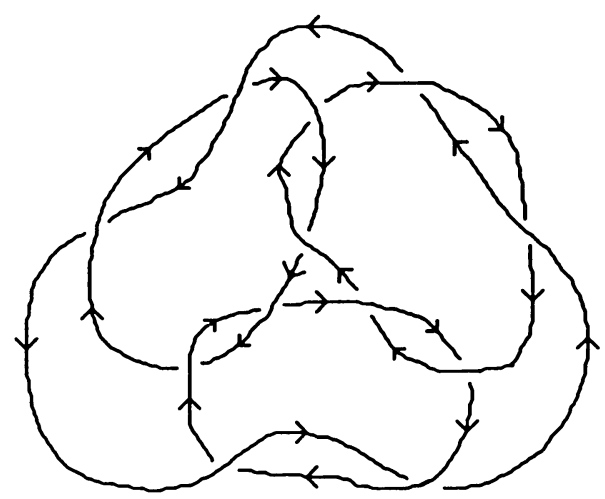

FIGURE 4

PROPOSITION 1. Let $G$ be a connected plane graph. Then for all nonzero numbers $x, y, z$ :

$$
P(D(G), x, y, z)=(y / z)^{|V(G)|-1}(-z / x)^{|E(G)|} t\left(G,-x / y, 1-\left(\left(x y+y^{2}\right) / z^{2}\right)\right) .
$$

PrOOF. By induction on the number of edges of $G$. The property is easy to check when $G$ has exactly one edge $(D(G)$ is the trivial knot if this edge is a bridge; if it is a loop, $P(D(G), x, y, z)=(y / z)+\left(y^{2} / x z\right)-(z / x)$ : see for instance [7, Remark (6), first example]. We now assume that $G$ has at least two edges.

Let $e$ be an edge of $G$. By applying property (ii) of the homfly polynomial to one of the crossings of $D(G)$ associated to $e$, we obtain an equation which we describe pictorially in Figure 5 (we have three diagrams which are identical outside the region which we have represented, and each diagram $L$ stands for the value of $P(L, x, y, z))$ :

Then property (iii) (invariance over isotopy classes) yields the "pictorial equation" described in Figure 6.

Now we consider three cases:

(a) The edge $e$ is neither a bridge nor a loop of $G$. Then clearly our pictorial equation can be interpreted as

$$
P(D(G), x, y, z)=-(y / x) P(D(G . e), x, y, z)-(z / x) P(D(G-e), x, y, z) .
$$

Note that $G . e$ and $G-e$ are connected. Hence, by our induction hypothesis:

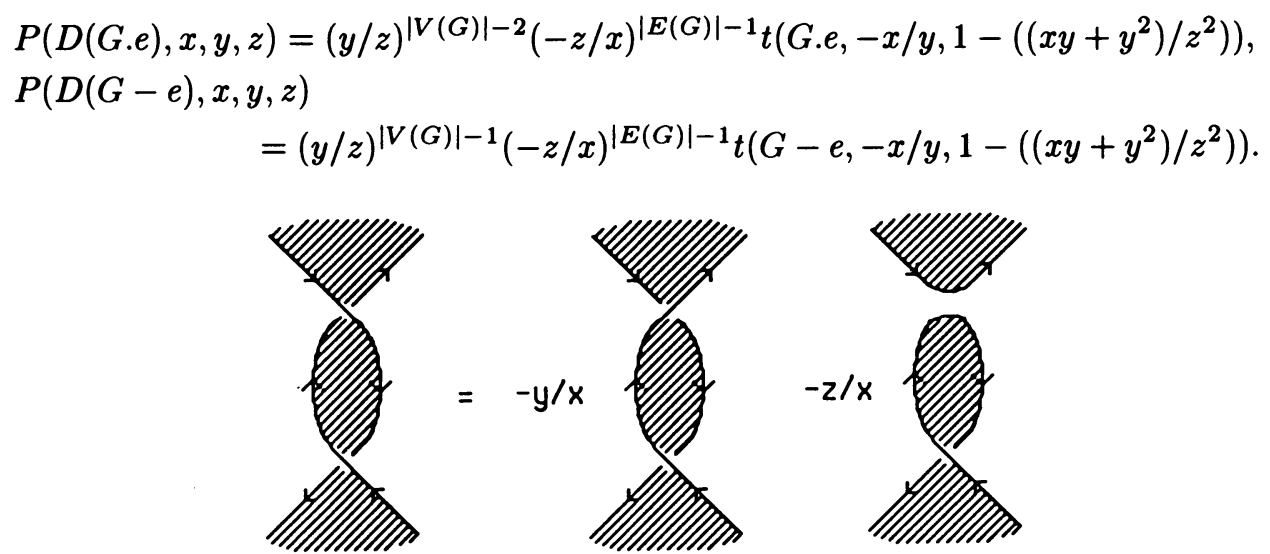

FIGURE 5 


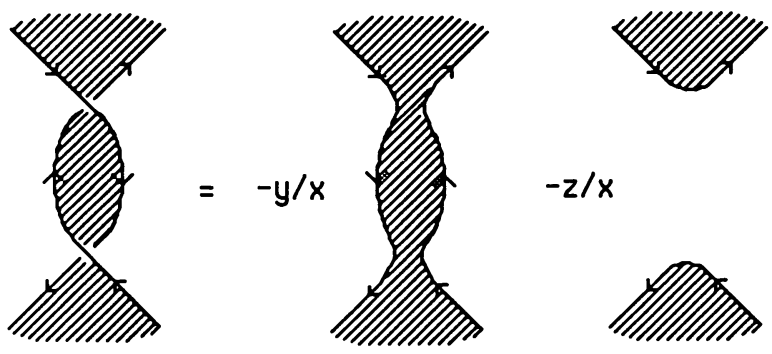

FIGURE 6

It follows that

$$
\begin{aligned}
P(D(G), x, y, z) & \\
=(y / z)^{|V(G)|-1}(-z / x)^{|E(G)|}(t(G . e & \left.-x / y, 1-\left(\left(x y+y^{2}\right) / z^{2}\right)\right) \\
& \left.+t\left(G-e,-x / y, 1-\left(\left(x y+y^{2}\right) / z^{2}\right)\right)\right)
\end{aligned}
$$

and property (2) of the Tutte polynomial yields the required result.

(b) The edge $e$ is a bridge of $G$. Then it is easy to see that $D(G)$ and $D(G . e)$ are isotopic. Consequently, $P(D(G), x, y, z)=P(D(G . e), x, y, z)$, and, by induction:

$$
P(D(G), x, y, z)=(y / z)^{|V(G)|-2}(-z / x)^{|E(G)|-1} t\left(G . e,-x / y, 1-\left(\left(x y+y^{2}\right) / z^{2}\right)\right) .
$$

The result now follows from property (3) of the Tutte polynomial.

(c) The edge $e$ is a loop of $G$. Coming back to our pictorial equation, we see that we may define diagrams $L^{\prime}, L^{\prime \prime}, L_{1}, L_{2}$ such that

$$
P(D(G), x, y, z)=-(y / x) P\left(L^{\prime}, x, y, z\right)-(z / x) P\left(L^{\prime \prime}, x, y, z\right),
$$

$L^{\prime}$ is split into $L_{1}$ and $L_{2}$, and $L^{\prime \prime}$ is the connected sum of $L_{1}$ and $L_{2}$.

It follows from property (iv) of the homfly polynomial that $P\left(L^{\prime}, x, y, z\right)=$ $-((x+y) / z) P\left(L^{\prime \prime}, x, y, z\right)$. Moreover $L^{\prime \prime}$ is clearly isotopic to $D(G-e)$. Hence

$$
P(D(G), x, y, z)=\left(\left(\left(x y+y^{2}\right) / x z\right)-(z / x)\right) P(D(G-e), x, y, z) .
$$

$G-e$ is connected and we may apply our induction hypothesis to obtain

$$
\begin{aligned}
& P(D(G-e), x, y, z) \\
& \quad=(y / z)^{|V(G)|-1}(-z / x)^{|E(G)|-1} t\left(G-e,-x / y, 1-\left(\left(x y+y^{2}\right) / z^{2}\right)\right) .
\end{aligned}
$$

Property (3) of the Tutte polynomial for the case of a loop is now easily seen to be equivalent to the required result. This completes the proof.

EXAMPLE. For $x=s, y=-1 / s, z=s^{1 / 2}-1 / s^{1 / 2}, P(D(G), x, y, z)$ is the Jones polynomial of $D(G)$ evaluated at $s$, which we denote by $V(D(G), s)$. Then Proposition 1 yields, for $s \neq 0, s \neq 1$ :

$$
\begin{aligned}
& V(D(G), s) \\
& \quad=\left(1 / s^{1 / 2}-s^{1 / 2}\right)^{|E(G)|-|V(G)|+1}(1 / s)^{|E(G)|+|V(G)|-1} t\left(G, s^{2},\left(s^{2}+1\right) /\left(s^{2}-s\right)\right)
\end{aligned}
$$

3. Consequences. We now study the possibility to use Proposition 1 for the evaluation of the Tutte polynomial of an arbitrary planar graph $G$. Since the Tutte polynomial of a graph is the product of the Tutte polynomials of its blocks, we may 
assume that $G$ is connected. We consider that $G$ is given an arbitrary embedding in the plane.

We may apply Proposition 1 to evaluate $t(G, u, v)$ whenever there exist nonzero numbers $x, y, z$ such that $-(x / y)=u, 1-\left(\left(x y+y^{2}\right) / z^{2}\right)=v$. This is not possible for $u=0$. For $u=1$, this is possible (with $x=-y$ and $z$ arbitrary) if and only if $v=1$. Finally for $u \neq 0, u \neq 1$ this is possible with $x=-u y, y= \pm((1-v) /(1-u))^{1 / 2} z$, unless $v=1$. Note that we must allow complex values for $x, y, z$ and that the vector $(x, y, z)$ is defined up to collinearity (this corresponds to the fact that the homfly polynomial is homogeneous of degree zero).

Thus we obtain the following equivalent form of Proposition 1.

PROPOSITION 2. For any connected plane graph $G$ :

(i) $t(G, 1,1)=(-1 / z)^{|E(G)|-|V(G)|+1} P(D(G), 1,-1, z)$ for every nonzero number $z$,

(ii) for $u \neq 0, u \neq 1, v \neq 1$ and $\varepsilon= \pm 1$

$$
\begin{aligned}
t(G, u, v)= & u^{|E(G)|}\left(\varepsilon(1-v)^{1 / 2} /(1-u)^{1 / 2}\right)^{|E(G)|-|V(G)|+1} \\
& \cdot P\left(D(G),-\varepsilon u(1-v)^{1 / 2}, \varepsilon(1-v)^{1 / 2},(1-u)^{1 / 2}\right) .
\end{aligned}
$$

We may interpret Proposition 2(i) as follows. $P(D(G), 1,-1,-z)$ is the Alexander-Conway polynomial of $D(G)$ evaluated at $z$ (see [5]), which we denote by $\nabla(D(G), z)$. On the other hand, $t(G, 1,1)$ is the number of spanning trees of $G$, that is, its complexity, which we denote by $c(G)$. Thus

COROLlaRY 3. For every connected plane graph $G$,

$$
\nabla(D(G), z)=z^{|E(G)|-|V(G)|+1} c(G) .
$$

It is an easy exercise to prove Corollary 3 using the "state model" devised by Kauffman for the Alexander-Conway polynomial [15].

Let us recall that if $G^{*}$ denotes the geometric dual of $G, t\left(G^{*}, u, v\right)=t(G, v, u)$. Then Proposition 2 allows us to evaluate $t(G, u, v)$ using the homfly polynomial of $D(G)$ or $D\left(G^{*}\right)$ for all $u, v$ such that $u=1$ iff $v=1$, except when $u=v=0$ which is a trivial case. Thus essentially the Tutte polynomial can be viewed as a link invariant. More precisely, using the algebraic definition of the homfly polynomial $[\mathbf{7}, \mathbf{1 1}]$, problems about the Tutte polynomial of planar graphs can be reformulated as problems in the braid groups, or as problems in suitable Von Neumann algebras.

For instance, let us consider the Potts model of statistical mechanics (see [2, Chapter 12]). The physical system under study is represented by a graph $G$ which we assume to be planar (physicists usually deal with "lattices" which are highly regular infinite graphs; these lattices can be viewed as "limits" of sequences of finite graphs). Each vertex of the graph $G$ can be in one of $q$ possible states, so that the possible states for $G$ itself can be described as colorings of the vertices with colors in $\{1, \ldots, q\}$. Two adjacent vertices interact when they are in the same state, and the interaction energy is then equal to a constant $-J$. Thus the energy $e(S)$ of a state $S$ is $-J|U(S)|$, where $U(S)$ is the set of edges unicolored by $S$. The partition function of the model is defined as $Z=\sum_{S} \exp (-e(S) / k T)$, where $k$ is Boltzmann's constant and $T$ is the temperature. Hence, defining $K=J / k T, v=\exp (K)-1$, we obtain

$$
Z=\sum_{S} \exp (K|U(S)|)=\sum_{S}(1+v)^{|U(S)|}=\sum_{S} \sum_{F \subseteq U(S)} v^{|F|}
$$


Clearly the number of states $S$ such that $F \subseteq U(S)$ is equal to $q^{c(F)}$, where as before $c(F)$ denotes the number of connected components of the graph formed on the vertices of $G$ by the edges of $F$. It follows that

$$
Z=\sum_{F \subseteq E(G)} v^{|F|} q^{c(F)} .
$$

Now the subgraph expansion for the Tutte polynomial of $G$ can be rewritten as

$$
t(G, x, y)=(x-1)^{-c(E(G))}(y-1)^{-|V(G)|} \sum_{F \subseteq E(G)}(y-1)^{|F|}((x-1)(y-1))^{c(F)}
$$

and, assuming that $G$ is connected, we obtain

$$
Z=v^{|V(G)|-1} q t(G, 1+(q / v), 1+v) .
$$

Then using Proposition 1 or 2 we may write in the general case

$$
Z=(q+v)^{|E(G)|}\left(-q^{-1 / 2}\right)^{|E(G)|-|V(G)|-1} P\left(D(G), q+v,-v, q^{1 / 2}\right) .
$$

Thus the (unsolved) problem of the computation of $Z$ for, say, a "grid graph" (a portion of the infinite square lattice) reduces to the computation of the homfly polynomial for a special kind of link diagram. It would be interesting to obtain a closed braid representation for these diagrams.

The coloring problem is just a special case of the study of the Potts model. Indeed for $v=-1$, the partition function $Z$ gives the number of colorings of the vertices of $G$ with no unicolor edges (this is the well-known chromatic polynomial of $G$ ). In this case we have

$$
Z=(q-1)^{|E(G)|}\left(-q^{-1 / 2}\right)^{|E(G)|-|V(G)|-1} P\left(D(G), q-1,1, q^{1 / 2}\right) .
$$

For instance the Four Color Theorem [1] is equivalent to the fact that $P(D(G), 3,1,2)$ is nonzero for every loopless connected plane graph $G$. Thus the following topics seem to be of interest.

(1) Can the Four Color Theorem be extended (possibly with a simpler proof!) via the study of $P(L, 3,1,2)$ for general links $L$ ?

(2) Can one characterize the closed braids which are isotopic to a link of the form $D(G)$ for some loopless plane graph $G$ ?

Finally we observe that there is clearly a polynomial-time algorithm which, given a planar graph $G$, will produce the associated link diagram $D(G)$; since the coloring problem for planar graphs is known to be $N P$-complete (see [8] for more details), it follows that the problem of the computation of the homfly polynomial is $N P$-hard. Intuitively speaking, this means that finding a polynomial-time algorithm for this computation is equivalent to a negative solution of the famous $P \neq N P$ conjecture.

\section{REFERENCES}

1. K. Appel and W. Haken, Every planar graph is four colorable, Part I; W. Haken, K. Appel, and J. Koch, Every planar graph is four colorable, Part II, Illinois J. Math. 21 (1977), 429-567.

2. R. J. Baxter, Exactly solved models in statistical mechanics, Academic Press, New York, 1982.

3. C. Berge, Graphes et hypergraphes, Dunod, Paris, 1974.

4. J. A. Bondy and U. S. R. Murty, Graph theory with applications, Macmillan, London, 1976.

5. J. H. Conway, An enumeration of knots and links and some of their algebraic properties, Computational Problems in Abstract Algebra, Pergamon Press, New York, 1970, pp. 329-358. 
6. J. R. Edmonds, Pictures of knots and construction of planar triangulations, Communication at the Third International Conference "Théorie des graphes et combinatoire," Marseille, June 1986.

7. P. Freyd, D. Yetter, J. Hoste, W. B. R. Lickorish, K. Millett, and A. Ocneanu, A new polynomial invariant of knots and links, Bull. Amer. Math. Soc. (N.S.) 12 (1985), 239-246.

8. M. R. Garey and D. S. Johnson, Computers and intractability: a guide to the theory of NPcompleteness, Freeman, San Francisco, Calif., 1979.

9. J. Hoste, A polynomial invariant of knots and links, Pacific J. Math. 124 (2) 1986, 295-320.

10. V. F. R. Jones, A polynomial invariant for knots via Von Neumann algebras, Bull. Amer. Math. Soc. (N.S.) 12 (1985), 103-111.

11. __ A new knot polynomial and Von Neumann algebras, Notices Amer. Math. Soc. 33 (1986), 219-225.

12. L. H. Kauffman, New invariants in the theory of knots, preprint.

13. _ State models and the Jones polynomial, Topology 26 (1987), 395-407.

14. _ Statistical mechanics and the Jones polynomial, preprint.

15. __ Formal knot theory, Math. Notes 30, Princeton Univ. Press, Princeton, N.J., 1983.

16. K. Murasugi, Jones polynomials and classical conjectures in knot theory, Topology 26 (1987), 187-194.

17. O. Ore, The four-color problem, Academic Press, New York, 1967.

18. H. N. V. Temperley and E. H. Lieb, Relations between the percolation and colouring problem and other graph-theoretical problems associated with regular plane lattices: some exact results for the percolation problem, Proc. Roy. Soc. London 322 A (1971), 251-280.

19. M. Thistlethwaite, A spanning tree expansion of the Jones polynomial, Topology 26 (1987), 297-309.

20. W. T. Tutte, A ring in graph theory, Proc. Cambridge Philos. Soc. 43 (1947), 26-40.

21. _ $\ldots$ contribution to the theory of chromatic polynomials, Canad. J. Math. 6 (1954), 80-91.

Laboratoire de Structures Discrètes, IMAG, BP53X, 38041 Grenoble CÉdeX, FRANCE 